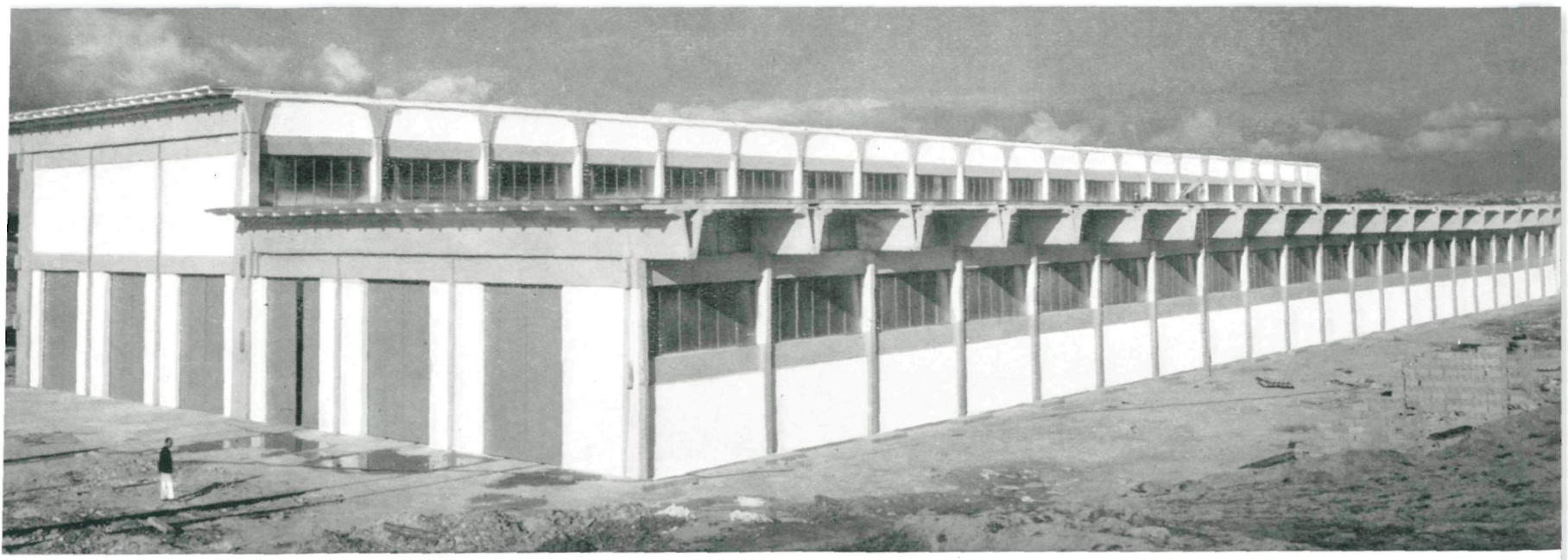

\title{
estructura de nave para el Talgo, en Aravaca
}

A. CORRAL LOPEZ-DORIGA y J. A. FERNANDEZ ORDOÑEZ, ingenieros

$138 \cdot 27$

\section{simopsis}

Sobre unos volúmenes dados, se proyecta una estructura a base de vigas canal en $\mathbf{V}$ pretensadas, resolviendo así el problema de desaguie que plantea una cubierta plana, en dos alturas, de $42,50 \mathrm{~m} \times 116,50 \mathrm{~m}$, consiguiendo al mismo tiempo con los elementos estructurales un mejor tratamiento del espacio. La estructura ha sido proyectada para conseguir un plazo de ejecución mínimo.

Las vigas en $\mathbf{V}$ se ejecutaron en una explanada contigua. Después se riparon, elevaron y colocaron en su posición definitiva por medio de un procedimiento original de montaje de la casa constructora. Los tramos entre vigas $V$ se cubrieron con viguetas pre-
tensadas, prefabricadas, de sección rectangular, sobre las que apoyaba la cobertura.

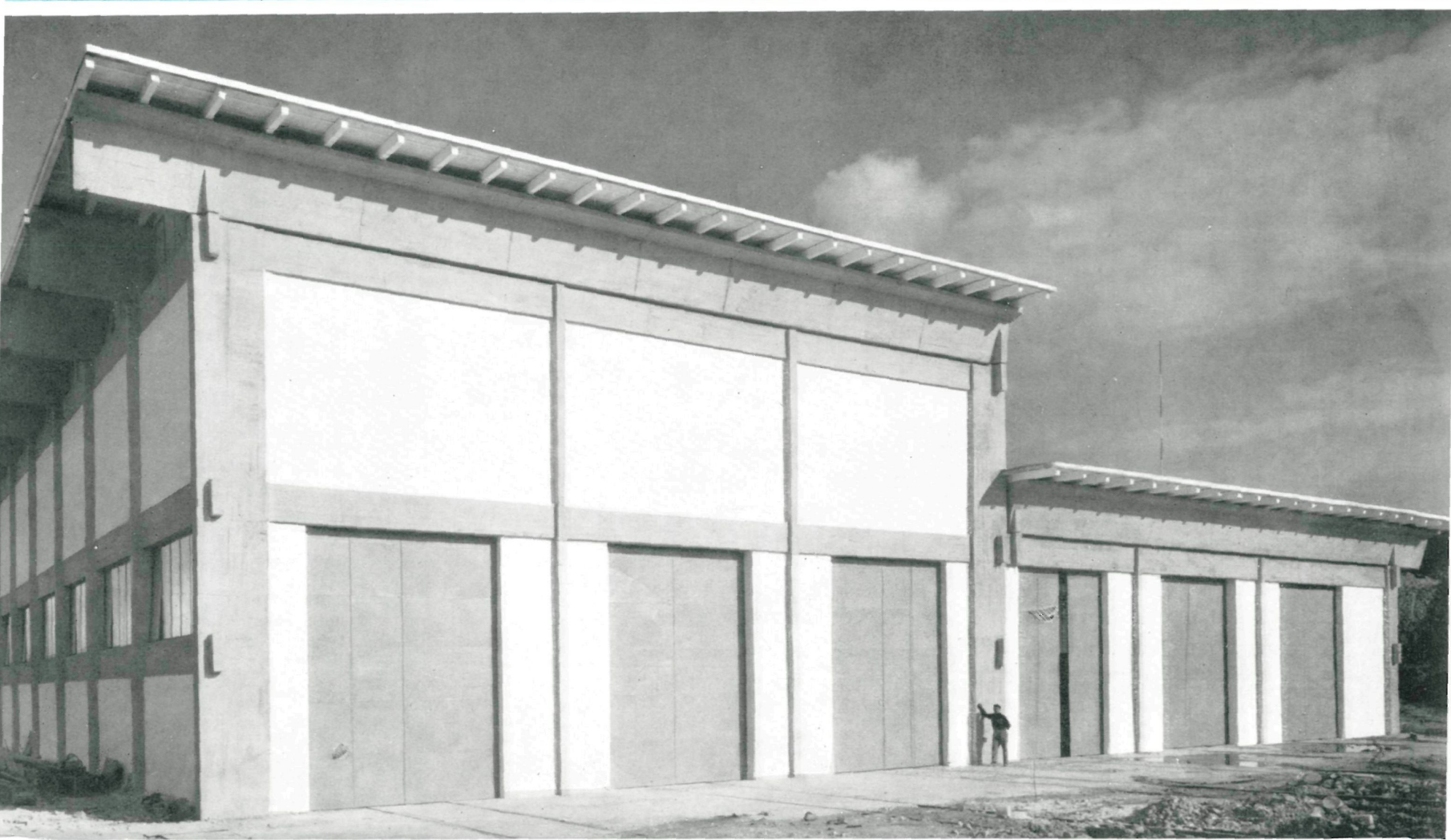




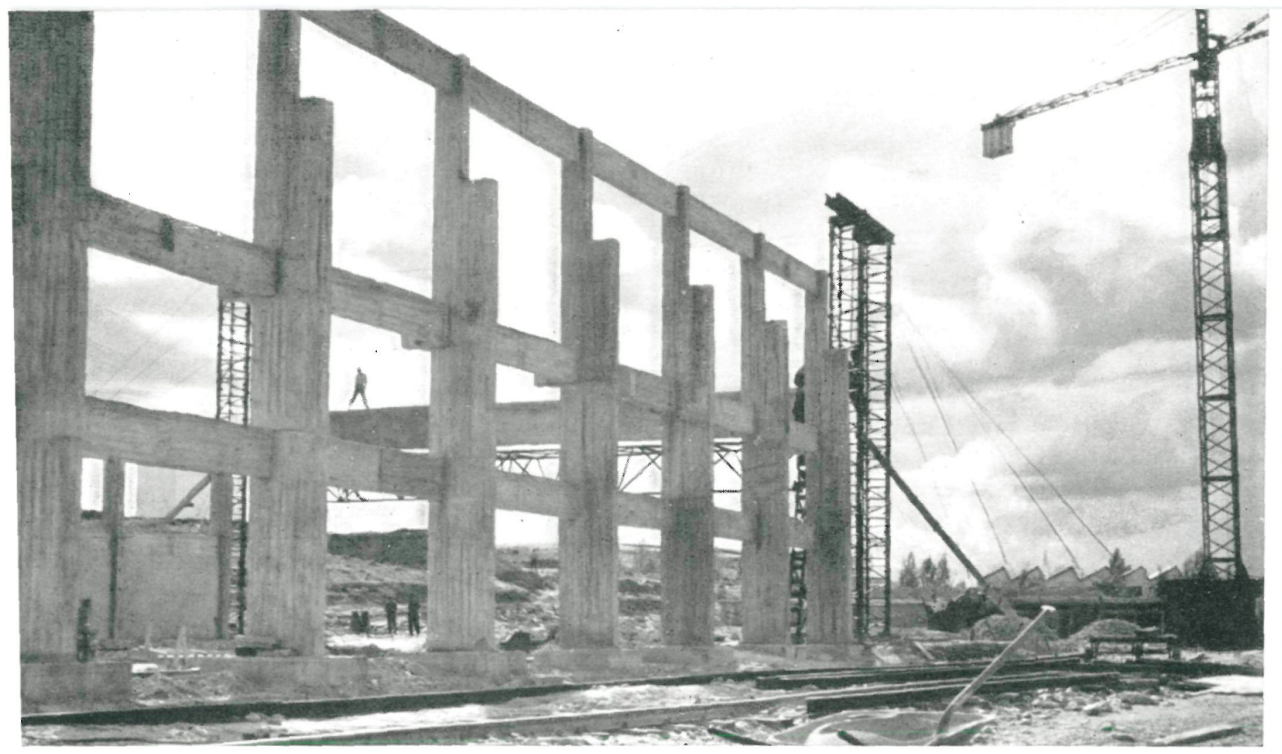

Hilera de pilares centrales.

Se proyectó y dirigić la construcción de la estructura de esta nave por encargo del arquitecto don Miguel Oriol. La sección de la nave estaba fijada y se componía de dos rectángulos de la misma base y de diferentes alturas; el más bajo de $6,00 \mathrm{~m}$ de altura y el contiguo de $10,50 \mathrm{~m}$, para dar cabida a un puente grúa de $15 \mathrm{t}$ de carga útil. La nave es prolongación de una construida anteriormente; al variar la modulación de luces transversales no estaba justificado conservar el tipo estructural de aquélla. Las distancias entre ejes de pórticos debía ser de $5,55 \mathrm{~m}$ y el número de éstos de 22.

Se proyectó la cubierta a base de unas vigas con seccićn en $\mathrm{V}$, que además de su función resistente cumplen la misión de evacuar el agua (viga canal), resolviendo así el problema que plantea el desagüe de una cubierta plana de estas dimensiones. Estas vigas son de hormigón pretensado, lo que, además de suponer una economía (sobre todo en cuanto al plazo de ejecución), evita el problema de su impermeabilización. pilar central
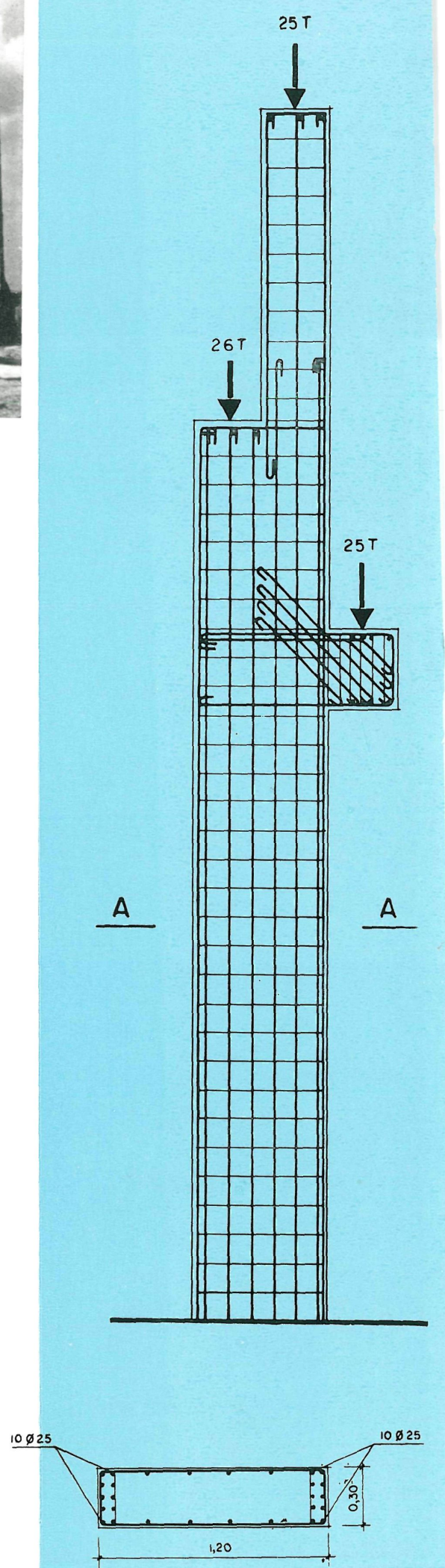

Sección $A-A$ 
El canto de éstas es de 1,30 m, que, aunque excesivo para los rebajamientos que permite el hormigón pretensado, no suponía un encarecimiento de las mismas, y, en cambio, permitía llegar a un tratamiento más armónico del espacio arquitectónico.

Las vigas en $\mathrm{V}$ pesaban $20 \mathrm{t}$ cada una, se ejecutaban dos por día y el ritmo de montaje por término medio fue de cinco vigas diarias. La armadura de pretensar está compuesta de seis cables $12 \varnothing 5 \mathrm{~mm}$, utilizándose el procedimiento Freyssinet de tesado y anclaje. De viga a viga, apoyadas en sus alas, van colocadas viguetas pretensadas prefabricadas de sección rectangular, sobre las que apoya el material de cobertura. El espacio que queda entre ellas se puede así aprovechar para proporcionar una iluminación difusa, valorando más la cubierta y permitiendo una mejor ventilación.

Fl pilar central es el que tiene mayores solicitaciones; es de $1,20 \mathrm{~m} \times 0,30 \mathrm{~m}$ en su parte inferior y de $0,60 \mathrm{~m} \times 0,30 \mathrm{~m}$ en la superior, viniendo determinadas estas dimensiones por la excentricidad de las cargas que actúan sobre él: cubierta superior, cubierta inferior y puente grúa. Las vigas de atado distribuyen el esfuerzo longitudinal del viento o bien el frenado del puente-grúa. Para el apoyo de las vigas de la nave inferior en el pilar central, y con objeto de no restarle espacio a ésta, se dispone de unas ménsulas en forma de cruz para que, además, la riga tenga estabilidad lateral.

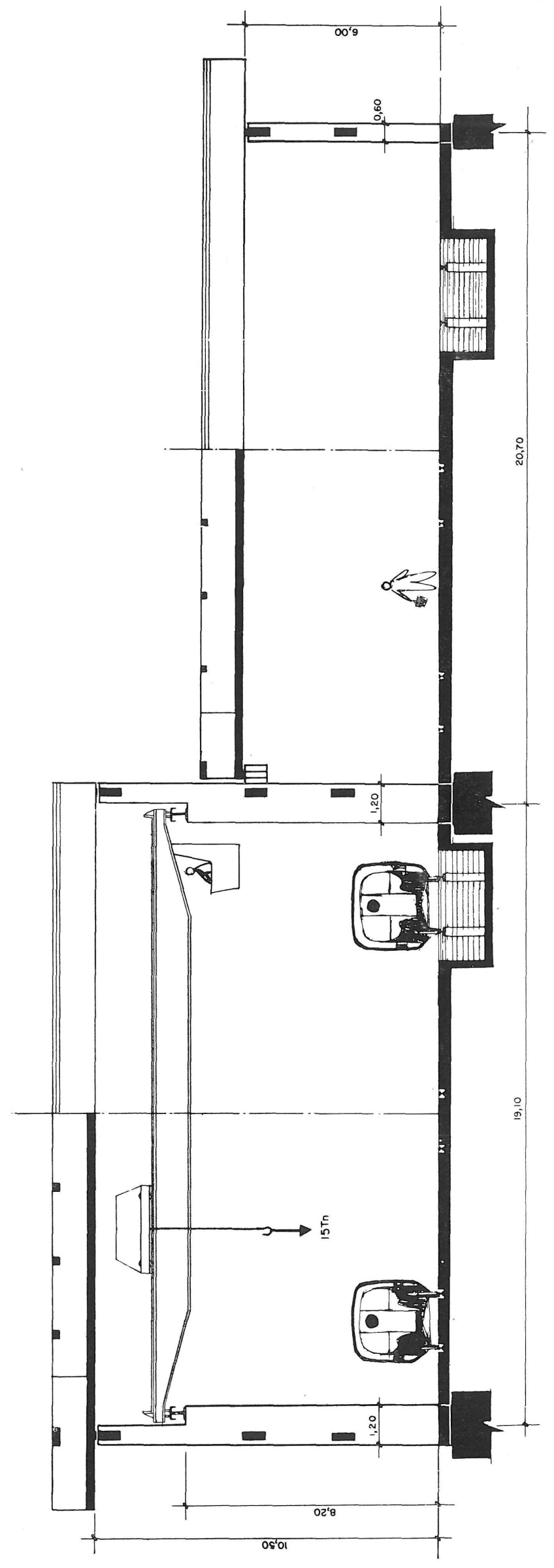



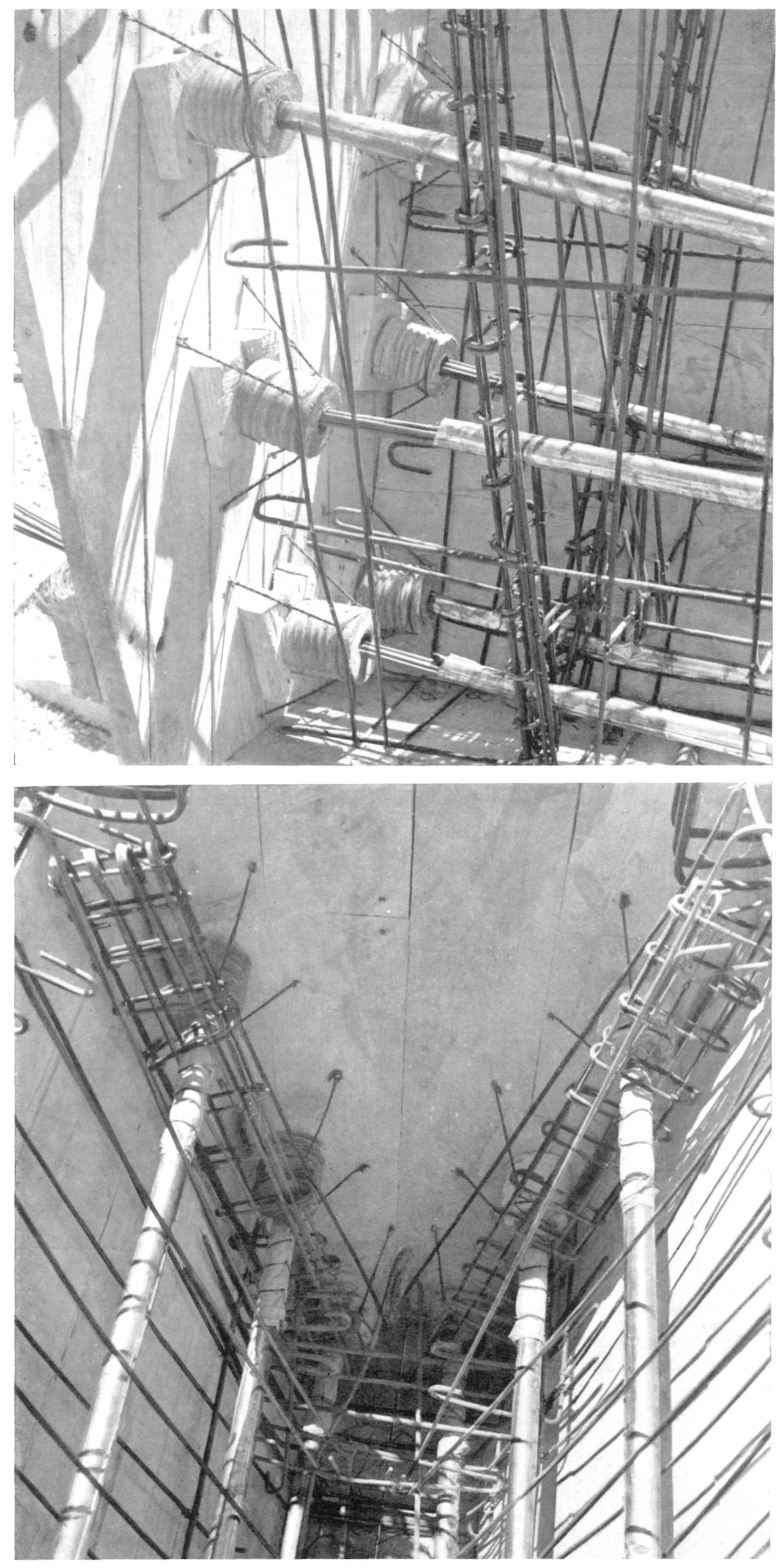

Los seis conos de anclaje fijados al encofrado, antes de cololas vainas y de poner en su sitio la armadura de zunchado.
Extremo de la armadura, conos de an je $y$ zunchos, a falta de colocar el encofrado interior. 

viga para colocarla sobre pórtico móvil.

La cubierta se prolonga a ambos lados en unos aleros que la definen esdizos de las vigas, de 1,85 metros de longitud, están resueltos en hormigón armado, para que sus cabezas presenten las dimensiones y espesores reales de la sección tipo y no se vean perturbadas por el aumento de sección necesario para los anclajes. Estos voladizos se armaron con los mismos alambres de pretensar que sobresalían de los anclajes.
Suspensión y elevación de una tructuralmente. Los vola-
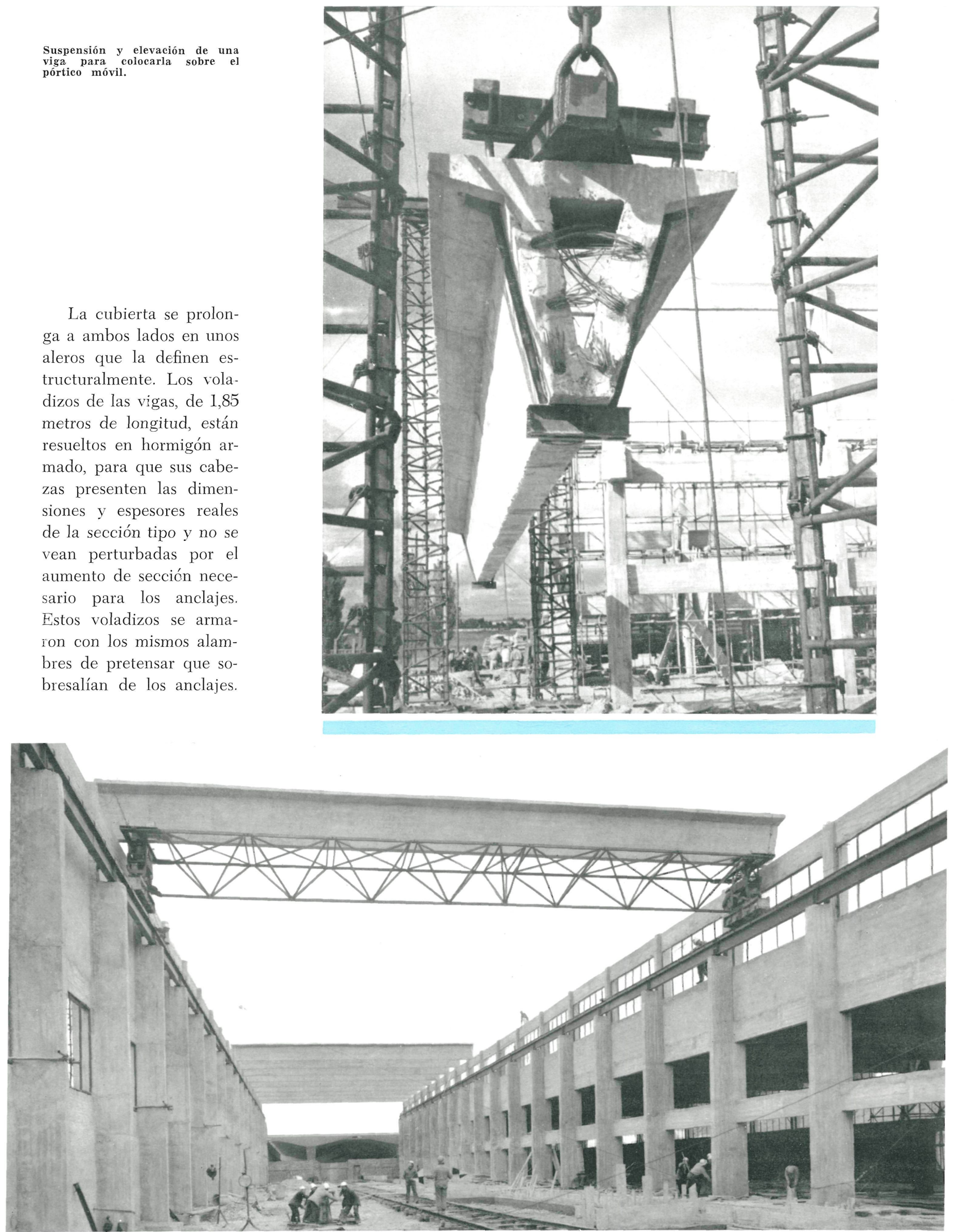

Traslación de una viga en la nave alta aprovechando la viga carril del puente-grúa. 

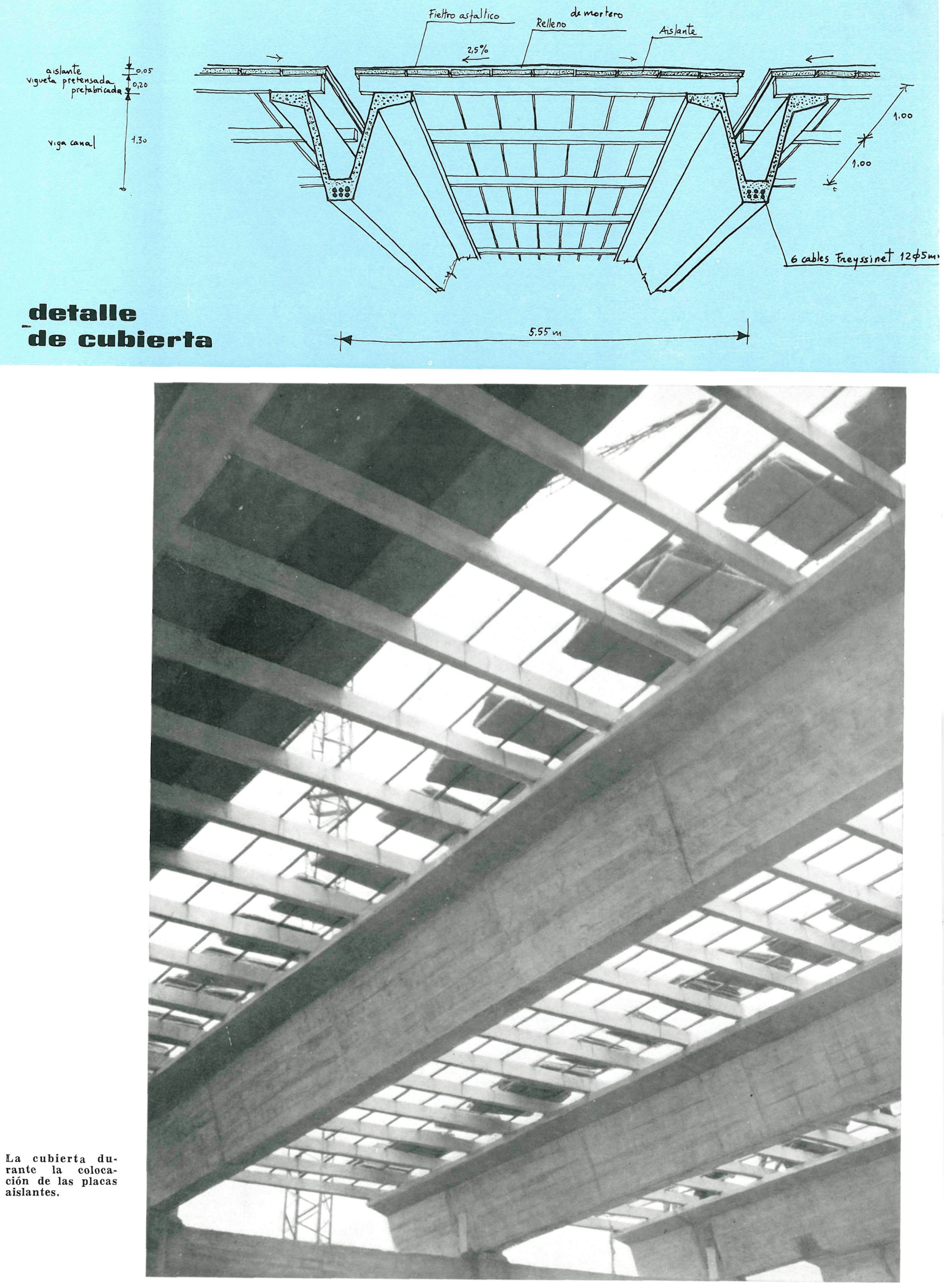

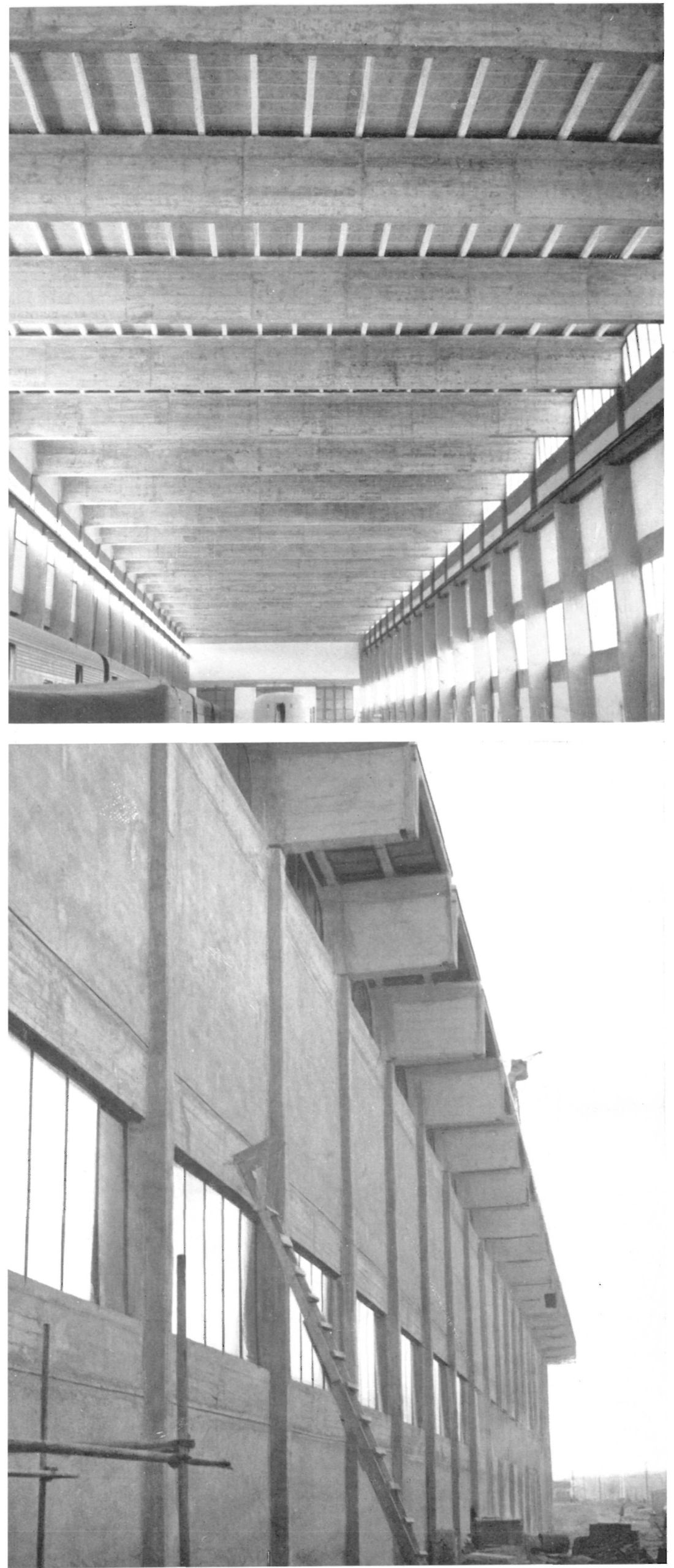

Interior de la cubierta de la nave alta.

Fachada norte.
Las 44 vigas en $\mathrm{V}$ se hormigonaron y pretensaron en una explanada contigua a la obra. Una vez terminadas se ripaban, apoyadas en sus extremos sobre dos bicicletas, hasta el primer pórtico; allí eran elevadas, suspendidas en sus extremos, por medio de dos torres y colocadas sobre un pórtico móvil que las trasladaba a su emplazamiento y, por medio de unos gatos, se dejaban en su posición definitiva.

En la nave alta, se utilizaba la viga carril del puente-grúa como camino de rodadura del pórtico móvil.

La duración de las obras de cimentacićn y superestructura fue de cuatro meses. La obra fue construida por la empresa Prefabricacićn Pesada y Pretensados, del grupo Agromán. 


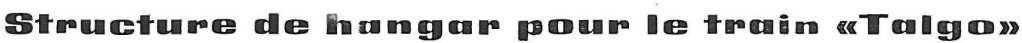

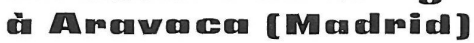

Alberto Corrai López-Dóriga et José Antonio Fernández Ordóñez, ingenieurs.

La structure de ce hangar, basée sur des poutres en $\mathrm{V}$ précontraintes, a été calculé pour des volumes déterminés. Ainsi a été résolu le problème d'écoulement posé par une couverture plate, de deux niveaux, de $42,50 \times 116,50 \mathrm{~m}$, tout en obtenant une meilleure utilisation des espaces pour une exécution en un minimum de temps.

Les poutres en $\mathbf{V}$ ont été exécutées sur une esplanade contiguë. Elles furent ensuite ripées, élevées et mises définitivement en place à l'aide d'un procédé original de montage de l'entreprise chargées de cet ouvrage. Les travées entre les poutres en $\mathbf{V}$ ont été couvertes de poutrelles précontraintes, préfabriquées, de section rectangulaire, sur lesquelles s’appuie la couverture.

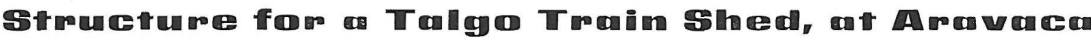

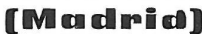

Alberto Corral Lopez Doriga and Jose Antonio Fernandez Ordoñez, engineers.

This structure was designed to cover a given sized shed. The main items were prestressed, $V$ shaped, channel beams. Their shape solved the difficulty of draining away water from a flat roof. The roof itself is constructed on two levels, and its area is 42.5 by $116.5 \mathrm{~m}$. These beams, moreover, have made it possible to make better use of the available space, and have also reduced to the minimum the construction time.

The $\mathbf{V}$ beams were built on a nearby flat ground. They were later slid, lifted and placed in their final position, with the aid of an original erection procedure adopted by the contractors. The spaces between the $V$ beams have been spanned with prefabricated, prestressed joists, of rectangular cross section, and on these the roofing material itself as been placed.

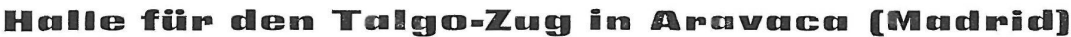

Alberto Corral López-Dóriga und José Antonio Fernández Ordóñez, Ingenieure.

Über einem gegebenen Raum wurde eine Halle mit Hilfe von vorgespannten Kanalträgern in V-Form entworfen. Letztere lösen gleichzeitig das Entwässerungsproblem, das ein flaches Dach in zwei verschiedenen Höhen von $42,50 \mathrm{~m} \times 116,50 \mathrm{~m}$ Fläche mit sich bringt. Gleichzeitig erreichte man auch durch die V-Träger eine bessere Raumgestaltung. Die Halle wurde so entworfen, dass sie in der kürzest möglichen Zeit erstellt werden konnte.

Die V-Träger wurden auf einem Platz neben dem Bau angefertigt und nach einer besonderen Einbaumethode der Baufirma in ihre endgültige Lage gebracht. Die Abschnitie zwischen den V-Trägern wurden mit kleineren rechteckigen vorgespannten und vorgefertigten Trägern überdeckt, auf denen das Dach ruht. 